NASA/TM-2001-211278

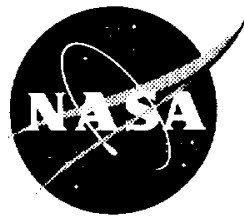

\title{
A Robust Design Methodology for Optimal Microscale Secondary Flow Control in Compact Inlet Diffusers
}

Bernhard H. Anderson

Glenn Research Center, Cleveland, Ohio

Dennis J. Keller

RealWorld Quality Systems, Rocky River, Ohio

Prepared for the

40th Aerospace Sciences Meeting and Exhibit

sponsored by the American Institute of Aeronautics and Astronautics

Reno, Nevada, January 14-17, 2002

National Aeronautics and

Space Administration

Glenn Research Center 
Available from

NASA Center for Aerospace Information 7121 Standard Drive

Hanover, MD 21076
National Technical Information Service 5285 Port Royal Road Springfield, VA 22100

Available electronically at http://gltrs.grc.nasa.gov/GLTRS 


\title{
A ROBUST DESIGN METHODOLOGY FOR OPTIMAL MICROSCALE SECONDARY FLOW CONTROL IN COMPACT INLET DIFFUSERS
}

\author{
Bernhard H. Anderson \\ National Aeronautics and Space Administration \\ Glenn Research Center \\ Cleveland, Ohio 44135 \\ Dennis J. Keller \\ RealWorld Quality Systems \\ Rocky River, Ohio 44116
}

\begin{abstract}
It is the purpose of this study to develop an economical Robust design methodology for micro-scale secondary flow control in compact inlet diffusers. To illustrate the potential of economical Robust Design methodology, two different mission strategies were considered for the subject inlet, namely (1) Maximum Performance, and (2) Maximum HCF Life Expectancy. The Maximum Performance mission maximized total pressure recovery while the Maximum HCF Life Expectancy mission minimized the mean of the first five Fourier harmonic amplitudes, i.e. "collectively" reduced all the harmonic 1/2 amplitudes of engine face distortion. Each of the mission strategies was subject to a low engine face distortion constraint, i.e. DC60 $\leq 0.10$, which is a level acceptable for commercial engines. For each of these missions strategies, an "Optimal Robust" (open loop control) and an "Optimal Adaptive" (closed loop control) installation was designed over a twenty degree angle-of-incidence range. The "Optimal Robust" installation used economical Robust Design methodology to arrive at a single design which operated over the entire angle-of-incident range (open loop control). The "Optimal Adaptive" installation optimized all the design parameters at each angle-of-incidence. Thus the "Optimal Adaptive" installation would require a closed loop control system to sense a proper signal for each effector and modify that effector device, whether mechanical or fluidic, for optimal inlet performance. In general, the performance differences between the "Optimal Adaptive" and "Optimal Robust" installation designs were found to be marginal. This suggests, however, that "Optimal Robust" open loop installation designs can be very competitive with "Optimal Adaptive" close loop designs. Secondary flow control in inlets is inherently robust, provided it is optimally designed. Therefore, the new methodology presented in this paper. combined array "Lower Order" approach to Robust DOE, offers the aerodynamicist a very viable and economical way of exploring the concept of Robust inlet design, where the mission variables are brought directly into the inlet design process and insensitivity or robustness to the mission variables becomes a design objective
\end{abstract}

\section{INTRODUCTION}

The current development strategy for combat airvehicles is directed towards reduction in the Life-Cycle Cost (LCC) with little or no compromise to air-vehicle performance and survivability. This strategy has been extended to the aircraft component level, in particular, the engine inlet diffuser system. One method to reduce inlet system LCC is to reduce its structural weight and volume. Consequently, advanced combat inlet configurations are being made more compact (or shorter) to achieve weight and volume (and LCC) reduction. However, compact $S$-duct diffusers, see figures (1) and (3), are characterized by high distortion and low pressure recovery, produced by extreme wall curvature and strong secondary flow gradients. These characteristics are further aggravated by maneuver conditions. Since survivability rather than aerodynamic performance often drives the inlet design, it is expected that the flow quality entering the turbine engine will present an additional challenging environment for both fan/compressor surge margin and aeromechanical vibration. Interest in High Cycle Fatigue (HCF) research by the US aerospace community has been spurred by discrepancies between the expected durability of engine components compared to that actually experienced in the field. Recognizing that inlet distortion is a forcing function for vibration in the fan components, methods for increasing HCF Life Expectancy can been combined with techniques for inlet recovery and engine face distortion management. Therefore, to enable acceptable performance levels in such advanced. compact inlet diffuser configurations, micro-scale secondary flow control (MSFC) methods are being developed to manage the recovery, distortion, and $\mathrm{HCF}$ aspects of distortion. (1)-(2)

One of the most difficult tasks in the design of MSFC installation for optimal inlet operation is arriving at the geometric placement, arrangement, number, size and orientation of the effector devices within the inlet duct to achieve optimal performance. These actuator devices can be either mechanical or fluidic. This task is complicated not only by the large number of possible design variables available to the aerodynamicist, but also by the number of decisions parameters that are brought into the design process. By including the HCF effects into the inlet design process, the aerodynamicist has a total of seven individual response variables which measure various aspects of inlet performance. They include the inlet total pressure recovery, the inlet total pressure recovery distortion at the engine face and the first five Fourier harmonic 1/2 amplitudes of distortion. Each of these responses needs to be either maximized, minimized, constrained or unconstrained while searching for the optimal combination of primary design variable values that satisfy the mission requirements. The design task is further complicated by the existence of hard-tocontrol factors which effect inlet performance, i.e. the mission variables. The mission variables that cause the off- 
(engine corrected weight flow), angle-of-incidence and angle-ofyaw. While the aerodynamicist does not know how the pilot is ultimately going to fly the aircraft, it is known how the mission variables effect inlet performance under wind tunnel conditions. Traditionally, tolerance or robustness to the mission variables was accomplished only after the parameter design was completed, usually by accepting whatever off-design performance was delivered by the newly designed inlet system. Numerical optimization procedures that have been successful with some aerodynamics problems give little assistance to designing robust inlets since they are point-design procedures, usually with only one decision parameter. However, there is a branch of statistical Design-of-Experiments (DOE) methodology which integrates both traditional Response Surface Methods (RSM) and Robustness considerations into a single optimization procedure. It presents new potential for further reduction of Total Quality Cosit over the traditional design approach.

Taguchi $^{(3)}$ coined the term Robust Parameter Design to describe an approach to industrial problem solving whereby the product variation is reduced by choosing levels of the control factors (design parameters) that make the product insensitive to the changes in the noise factors that represent sources of variations. These noise factors in industrial design are often the environmental variables, such as temperature and humidity conditions, properties of the material, and product aging. In some applications, they measure how the consumer uses or handles the product. In the aerodynamic design of inlet systems, there is a analogous situation to the industrial design problem. As mentioned above, the design of inlet systems is usually accomplished at the cruise condition (the on-design condition) while variations from the cruise condition are considered as an off-design penalty. Because the mission variables cause variation from on-design performance, they can be identified with the noise factors or environmental variables in the analogous industrial design problem. Likewise, how the pilot flies the aircraft can be identified with how the consumer uses or handles the product. In the industrial problem, the researchers must be able to control the environmental variables in a laboratory environment, even though they cannot be controlled at the production level or in the field. Likewise, the aerodynamic researcher can indeed control the mission variables in the wind tunnel environment, however these variables cannot be controlled in flight (in the field). By making the analogy between the industrial design problem and the aerodynamic design problem, Robust Parameter Design methods developed for industrial problem solving can be adapted to the design of inlet systems, and in particular, design of micro-scale secondary flow control installations for such inlet systems.

Much has been written and said about the contribution of Genichi Taguchi to the vastly important area of Product Quality Enhancement. However, much controversy surrounds Taguchi"s methodology among statisticians. Many statisticians have pointed out the apparent flaws in the Taguchi approach. However, it suffices to say the importance of Taguchi's contributions lies in the idea that process or product sensitivity to its environment can be incorporated into the optimal statistical Design-of-Experiment and subsequent analysis of data. To the aerodynamicist, it represents a quantum leap in the area of inlet design. For the first time, the mission variables can be directly introduced into the inlet design processes. The inlet system can now be designed to operate with optimal performance over a range of specified mission variables. Rigorous application of Taguchi's Robust Parameter Design method may not be optimal in the design of micro-scale secondary flow installations for inlet systems because it can mask information vital to the aerodynamicist. However, the important aspects surrounding Taguchi's approach to Robust Parameter Design can and have been incorporated into an alternate approach. i.e. adopted to the inlet design problem. This is the subject of this report. The present methodology is a more standard method of statistical Design-of-Experiments, but with a unique data analysis phase. It provides a good working strategy for aerodynamic systems. Further, this method overcomes one of the basic hardships of Taguchi's method. namely the lack of economy in the experimental design plan.

Two different mission strategies were considered for the subject inlet, namely (1) Maximum Performance, and (2) Maximum HCF Life Expectancy. The Maximum Performance mission maximized total pressure recovery while the Maximum HCF Life Expectancy mission minimized the mean of the first five Fourier harmonic 1/2 amplitudes, i.e. "collectively" reduced all the Fourier harmonic 1/2 amplitudes of engine face distortion. Each of the mission strategies was subject to a low engine face distortion constraint, i.e. DC60 $\leq 0.10$, which is a level acceptable for commercial engines. For each of these missions strategies, an "Optimal Robust" (open loop control) and an "Optimal Adaptive" (closed loop control) installation was designed over a twenty degree angle-of-incidence range. The "Optimal Robust" installation arrived at a single MSFC installation which operated over the entire angle-of-incident range (open loop control). The "Optimal Adaptive" installation optimized all the design parameters at each angle-of-incidence. Thus the "Optimal Adaptive" installation would require a closed loop control system to sense a proper signal for each effector and modify that effector device, whether mechanical or fluidic, for optimal inlet performance. For each mission strategy, i.e. Maximum Performance and Maximum HCF Life Expectancy, the two approaches to secondary flow control installation design were compared for the simultaneous management of inlet total pressure recovery, engine face distortion, and the first five Fourier harmonic $1 / 2$ amplitudes of distortion. The angle-of incidence range was the Taguchi noise or environmental variable over which each optimal installation had to be robust.

\section{NOMENCLATURE}

$\begin{array}{ll}\text { AIP } & \text { Aerodynamic Interface Plane } \\ \text { c } & \text { Effector Chord Length } \\ \text { CCF } & \text { Central Composite Face-Centered } \\ \text { CFD } & \text { Computational Fluid Dynamics } \\ \text { D } & \text { Engine Face Diameter } \\ \text { DC60 } & \text { Circumferential Distortion Descriptor } \\ \text { DOE } & \text { Design of Experiments } \\ \text { h } & \text { Effector Blade Height } \\ \text { HCF } & \text { High Cycle Fatigue } \\ \text { Fi/2 } & \text { ith Fourier Harmonic 1/2 Amplitude } \\ \text { FM/2 } & \text { Mean Fourier Harmonic 1/2 Amplitude } \\ \text { L } & \text { Inlet Diffuser Length } \\ \text { LCC } & \text { Life Cycle Costs } \\ \text { MSFC } & \text { Micro-Scale Secondary Flow Control } \\ \text { Mt } & \text { Inlet Throat Mach Number } \\ \text { n } & \text { Number of Effector Vanes per Band } \\ \text { PFAVE } & \text { Inlet Total Pressure Recovery } \\ \text { R } & \text { Inlet Throat Radius } \\ \text { Re } & \text { Reynold Number per ft. } \\ \text { RSM } & \text { Response Surface Methodology } \\ \text { Y } & \text { Generalized Response Variable }\end{array}$




$$
\begin{array}{ll}
\alpha & \text { Inlet Angle-of-Incidence } \\
\beta & \text { Effector Vane Angle-of-Incidence } \\
\beta_{i j} & \text { Coefficients in the Regression Model } \\
\gamma & \text { Inlet Angle-of-Yaw }
\end{array}
$$

\section{RESULTS AND DISCUSSION}

The basic problem of experimental and CFD analysis design is deciding what pattern of test cases will best reveal aspects of the situation of interest. For that reason, the overall objectives of the study become very important. In the present study, three objectives were considered important, namely: (1) to determine the design characteristic (i.e. factor interactions) of multi-installation. micro-scale secondary flow control configurations, (2) to establish the ability of MSFC to manage the aeromechanical effects of engine face distortion, and (3) to evaluate the effectiveness of this new methodology for "open loop" microscale secondary flow installations over an angle-of-incidence range in comparison to fully adaptive "closed loop" designs. The first two objectives of this overall study on micro-scale flow control are covered by Anderson and $\operatorname{Keller}^{(4)}$, while the third objective is covered in detail in Anderson and $\mathrm{Keller}^{(5)}$. A third report in this series by Anderson and Keller ${ }^{(6)}$ evaluates the impact of rake geometry, specifically the number of rake arms, on the measurement errors associated with estimating the first five Fourier harmonic $1 / 2$ amplitude of engine face distortion. This paper covers the economical form of Robust Design methodology for micro-scale secondary flow installation design that appears in Anderson and Keller ${ }^{(5)}$.

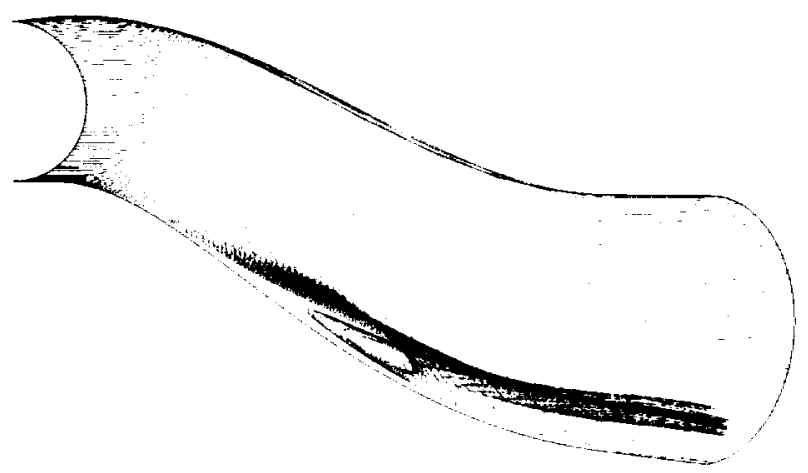

Figure (1): Particles traces showing the vortex liftoff (separation) within the DERA/M2129 inlet S-duct, $\mathrm{Mt}=0.70, \mathrm{Re}=$ $4.0 \times 10^{6} / \mathrm{ft}$., $\alpha=0.0^{\circ}$.

\section{Design of the Experiment}

The basic inlet flowpath chosen for this study featured a compact ( $L / D=3.0$ ), two turn, or $S$-duct inlet diffuser, Figure (1). This $\mathrm{S}$-duct was defined by AGARD FDP Working Group 13 Test Case 3, Willmer, Brown and Goldsmith ${ }^{(7)}$, and was dubbed the DERA/M2129 inlet. Traditionally, this type of compact inlet duct would be excluded from design consideration since it is characterized by severe wall curvature that induces strong secondary flows. These strong secondary flow can cause a flow separation called vortex lift-off. See Figure (1). This type of 3D flow separation results in severe total pressure losses and severe engine face distortion. Figure (2) presents the engine face total pressure recovery contours and secondary flow velocity vectors for the DERA/M2129 inlet S-duct at a throat Mach number of 0.70 . A vortex pair is dominant in the engine face flow field and this was accompanied by very severe engine face total pressure distortion.

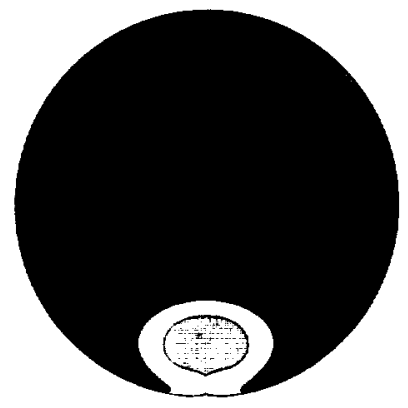

(a) Total Pressure

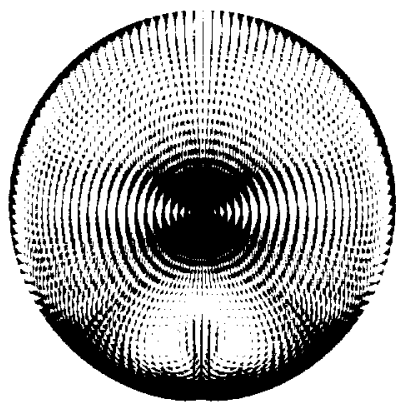

(b) Velocity Vectors
Figure (2): Baseline engine face solution, $M t=0.70, \operatorname{Re}=4.0 x$ $10^{6} / \mathrm{ft}$., $\alpha=0.0^{0}$.

To manage the flow in the DERA/M2129 inlet S-duct, a three-band installation arrangement of micro-scale effectors was placed in the upstream section near the inlet throat. See Figures (3) and (4). These micro-scale effectors were simple vanes, the largest height being about the average height of the momentum layer at the location of band (3), or about $2.0 \mathrm{~mm}$. The purpose of these simple vanes was to create a set of co-rotating vortices that will quickly merge to form a thin layer of secondary flow that will counter the formation of the passage vortex pair ${ }^{(1)}$. Since the height of the vane effectors were limited to $2.0 \mathrm{~mm}$, a multi-band arrangement was chosen to investigate the possibility of enhancing the effect of the individual vanes by adding more bands of effectors.

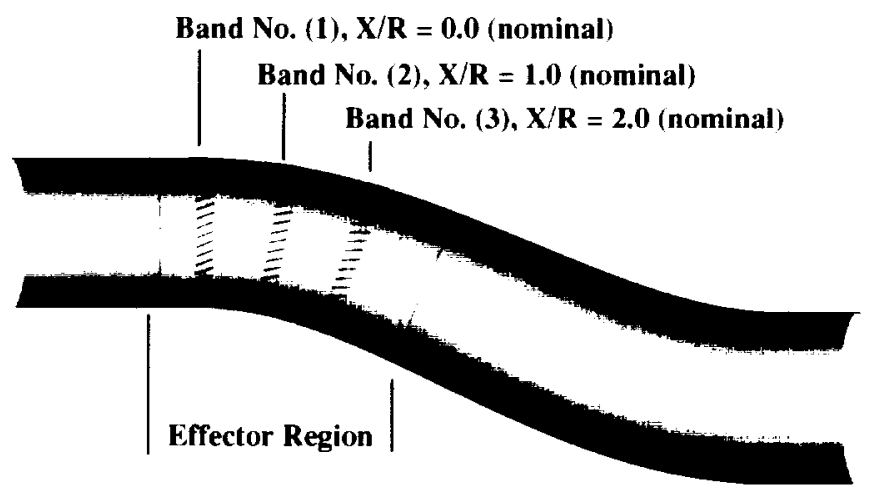

Figure (3): Location of effector region within the DERA/ M2129 inlet S-duct.

The spacing between the bands is critical since interaction will occur between respective bands of effector units. The first band was placed at the inlet throat station, $X / R=0.0$, while the second and third bands of effector vanes were placed nominally at $X / R=1.0$ and at $X / R=2.0$ respectively. See Figure (3). Nominally, the spacing between the effector vanes was $\Delta \mathrm{X} / \mathrm{c}=$ 
4.0 , i.e. about four effector chord lengths as measured between the half chord stations. See Figure (4).

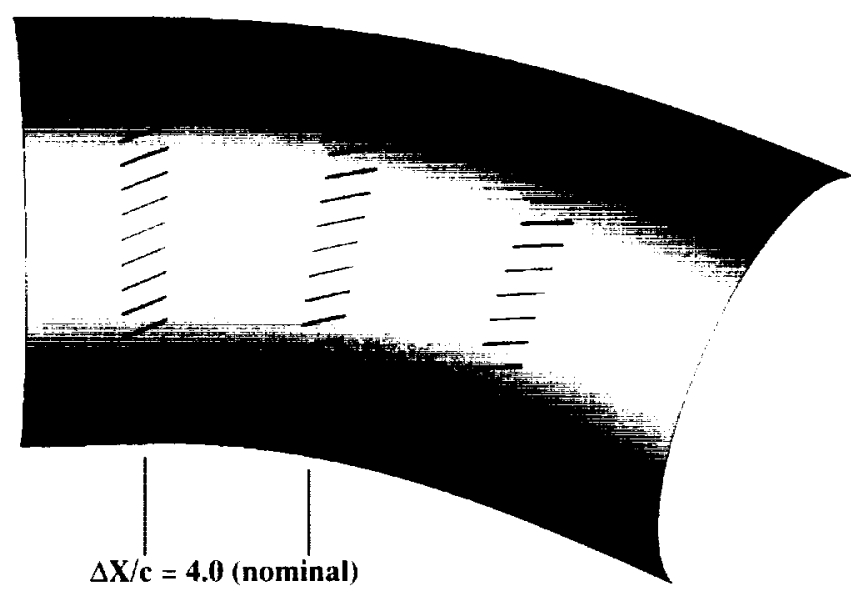

Figure (4): Micro-scale vane actuator arrangement within effector region.

The DOE approach followed directly from the three objectives previously stated and was reflected in the layout of the design factors listed in Table (1). The design variables (factors) were the effector vane heights $(\mathrm{mm})$ in the three upstream installation $h_{1}, h_{2}$, and $h_{3}$, and the inlet angle-of-incidence $\alpha$. The effector vane heights were changed independently and, therefore, constituted three independent variables. Strictly speaking, the inlet angle-of-incidence was a mission variable and was, therefore, one of the noise factors that belonged with the environmental variables, i.e. the outer array in the traditional Taguchi-style DOE design. In this study, however, the angle-of-incidence was introduced into the statistical design matrix with the control factors which allowed greater economy than the traditional Taguchi approach.

\begin{tabular}{|c|c|}
\hline Factors & Range \\
\hline Installation Vane Height $(\mathrm{mm}), \mathrm{h}_{1}$ & 0.0 to 2.0 \\
\hline Installation Vane Height $(\mathrm{mm}), \mathrm{h}_{2}$ & 0.0 to 2.0 \\
\hline Installation Vane Height $(\mathrm{mm}), \mathrm{h}_{3}$ & 0.0 to 2.0 \\
\hline Inlet Angle-of-Incidence, $($ degs.), $\alpha$ & 0 to 20.0 \\
\hline
\end{tabular}

Table (1): Factors which establish the DOE design matrix.

The robust nature of the angle-of-incidence was investigated during the analysis of the data phase. Table (2) shows the variables that were held constant during this study. The number of microscale vane effectors $n_{i, i}=1,3$ was held fixed at 24 in the half-plane, and were spaced symmetrically around the inlet half-periphery. Each vane effector was set at a geometric angle-of-incidence $\boldsymbol{\beta}_{\mathrm{i}}$, $\mathrm{i}=1,3$ of $24.0^{\circ}$. In addition, the throat Mach Number (Mt), Rey- nolds number $(\mathrm{Re})$, and the angle-of-yaw $(\gamma)$ were set constant at $0.70,4.0 \times 10^{6} / \mathrm{ft}$ and $0.0^{\circ}$ respectively for this investigation.

\begin{tabular}{|c|c|}
\hline Variable & Value \\
\hline Number of Effectors Units, $\mathrm{n}_{\mathrm{i}, \mathrm{i}=1,3}$ & 24 \\
\hline Vane Angle-of-Incidence, $\left(\right.$ degs.), $\boldsymbol{\beta}_{\mathrm{i}, \mathrm{i}=1, \mathbf{3}}$ & $24.0^{\circ}$ \\
\hline Installation Chord Length $(\mathrm{mm}), \mathbf{c}_{\mathbf{1}, \mathrm{i}=1,3}$ & 16.0 \\
\hline Throat Mach Number, $\mathrm{Mt}$ & 0.700 \\
\hline Reynolds Number, $x 10^{6} / \mathrm{ft}$. & 4.0 \\
\hline Inlet Angle-of-Yaw, $($ degs.), $\gamma$ & $0.0^{0}$ \\
\hline
\end{tabular}

Table (2): Variables held constant.

Table (3) displays the response variables for this study. They were the inlet total pressure recovery (PFAVE), the engine face distortion (DC60), and the first five Fourier harmonic $1 / 2$ amplitudes of engine face distortion (F1/2, F2/2, F3/2, F4/2, and F5/2).

\begin{tabular}{|c|c|}
\hline Design Responses & Term \\
\hline Engine Face Total Pressure Recovery & PFAVE \\
\hline Engine Face Distortion & DC60 \\
\hline 1st Fourier Harmonic 1/2 Amplitude & F1/2 \\
\hline 2nd Fourier Harmonic 1/2 Amplitude & F2/2 \\
\hline 3rd Fourier Harmonic 1/2 Amplitude & F3/2 \\
\hline 4th Fourier Harmonic 1/2 Amplitude & F4/2 \\
\hline 5th Fourier Harmonic 1/2 Amplitude & F5/2 \\
\hline
\end{tabular}

Table (3): DOE design response.

The DOE strategy selected was a Central Composite Face-Centered (CCF) DOE plus an additional experiment of special interest to the investigator. This strategy resulted in 26 unique experimental CFD cases that are shown in Table (4). Notice that these DOE cases covered a substantial range of possible flow situations over a wide range of angle-of-incidences from $0.0^{\circ}$ to $20.0^{\circ}$. This particular DOE, like most DOE strategies, varied more than one factor at a time. Further, this layout of 26 cases permitted the estimation of both linear and curvilinear effects as well as interactive or synergistic effects among the DOE factors. This is very important in the study of secondary flow control since very strong interaction effects can develop between separate bands of micro-scale effectors. This CCF DOE strategy is superior to the traditional approach where only changing one variable at a time does not permit the estimation of factor interactions. It is also more economical at 26 runs than a full factorial approach where the number of experiments would be $3^{4}$ or 81 
separate CFD cases. It is also more economical than a comparable Taguchi approach requiring $3 \times 15=45$ runs. Each of the 26 cases in Table (4) were run with a Reynolds-averaged NavierStokes code ${ }^{(9)}$ that allowed for numerical simulation of microvane effectors without the need to physically embed the vane effectors within the CFD grid structure.

\begin{tabular}{|c|c|c|c|c|}
\hline Config. & $h_{1}$ & $h_{2}$ & $\mathbf{h}_{3}$ & $\alpha$ \\
\hline nvg501 & 0.0 & 0.0 & 0.0 & 0.0 \\
\hline nvg502 & 2.0 & 0.0 & 0.0 & 0.0 \\
\hline nvg503 & 0.0 & 2.0 & 0.0 & 0.0 \\
\hline nvg504 & 2.0 & 2.0 & 0.0 & 0.0 \\
\hline nvg505 & 0.0 & 0.0 & 2.0 & 0.0 \\
\hline nvg506 & 2.0 & 0.0 & 2.0 & 0.0 \\
\hline nvg507 & 0.0 & 2.0 & 2.0 & 0.0 \\
\hline nvg508 & 2.0 & 2.0 & 2.0 & 0.0 \\
\hline nvg537 & 0.0 & 0.0 & 0.0 & 20.0 \\
\hline nvg538 & 2.0 & 0.0 & 0.0 & 20.0 \\
\hline nvg539 & 0.0 & 2.0 & 0.0 & 20.0 \\
\hline nvg540 & 2.0 & 2.0 & 0.0 & 20.0 \\
\hline nvg541 & 0.0 & 0.0 & 2.0 & 20.0 \\
\hline nvg542 & 2.0 & 0.0 & 2.0 & 20.0 \\
\hline nvg543 & 0.0 & 2.0 & 2.0 & 20.0 \\
\hline nvg544 & 2.0 & 2.0 & 2.0 & 20.0 \\
\hline nvg527 & 0.0 & 1.0 & 1.0 & 10.0 \\
\hline nvg528 & 2.0 & 1.0 & 1.0 & 10.0 \\
\hline nvg529 & 1.0 & 0.0 & 1.0 & 10.0 \\
\hline nvg530 & 1.0 & 2.0 & 1.0 & 10.0 \\
\hline nvg531 & 1.0 & 1.0 & 0.0 & 10.0 \\
\hline nvg532 & 1.0 & 1.0 & 2.0 & 10.0 \\
\hline nvg533 & 1.0 & 1.0 & 1.0 & 0.0 \\
\hline nvg551 & 1.0 & 1.0 & 1.0 & 20.0 \\
\hline nvg533 & 1.0 & 1.0 & 1.0 & 10.0 \\
\hline nvg519 & 0.0 & 0.0 & 0.0 & 10.0 \\
\hline
\end{tabular}

Table (4): Central Composite Face-Centered design (plus one additional case), “Lower Order" Robust Design methodology.
For the present study, however, the individual vanes were incorporated into the half-plane grid structure, and the appropriate boundary conditions applied. The half-plane grid structure was composed of three blocks: an upstream block, a effector section containing the micro-vanes, and a downstream block. See Figures (3) and (4). The computational half-plane grid varied in total number of mesh points from about 750.00 to $1,500,000$ depending on the micro-vane configuration. All CFD calculations were accomplished assuming half-plane symmetry. It was important to investigate the interactions between the separate effector bands without using the vane model in the code, so that proper band interaction could be established. This also established a set of baseline validation data to further verify the vane effector model in the Navier-Stokes code ${ }^{(9)}$ for multi-band flow control design concepts.

To introduce an angle-of-incidence ( $\alpha$-disturbance) into the flow analysis, the condition was imposed that the initial station have an angle-of-incidence component that approximated the measured angle-of-incidence flow field ${ }^{(10)}$. Even though introducing an $\alpha$-disturbance into the flow field is not rigorous, it provides a remarkable good approximation in comparison to experimental flow field. The overall intent of introducing an $\alpha$ disturbance into the flow field in this manner was to economically determine the degree of tolerance of the MSFC installation design to angle-of-incidence.

\section{Harmonic Analysis of Distortion}

The overall methodology used to obtain the harmonic content of inlet distortion was first proposed by Ludwig ${ }^{(1)}$ and is currently in use at the Williams International Corporation. This methodology is characterized by the use of radial weighting factors applied to the total pressure rake measurements. The radial weighting factors are shown in Table (5). These radial weighting

\begin{tabular}{|c|c|}
\hline Ring Number & Radial Weighting Coefficient \\
\hline 1 & 0.05651 \\
\hline 2 & 0.14248 \\
\hline 3 & 0.21077 \\
\hline 4 & 0.26918 \\
\hline
\end{tabular}

Table (5): Radial weighting coefficients applied to the total pressure rake measurements.

factors compress the rake information to a single radius ring of data samples, where the number of data samples corresponds to the number of arms of the measurement rake. A separate study was initiated by Anderson and $\operatorname{Keller}^{(6)}$ to evaluate the impact of rake geometry (specifically the number of rake arms) on the measurement error associated with estimating the first five Fourier harmonic 1/2 amplitudes of engine face distortion. As a result of that study, the rake and methodology chosen for this study was the 80 -probe clocked rake because it provided the lowest error in estimating the first five Fourier harmonic 1/2 amplitudes of engine face distortion. Using the AIP instrumentation locations for the 80 -probe (virtual) rake, the 26 CFD solutions were interpolated at each of the probe positions shown in Figure (5a). The 
span-weighted average total pressure was calculated for the 80 probe rake by multiplying the probe total pressure by the spanweighted coefficients from Table (5), and adding the results over the five probes of the rakes to form a single radius ring of data samples.

Since the rake at the engine face was "clocked", a complete set of "repeats" was generated at each experimental run in Table (4). From the engine face patterns at each of the 10 clocking angles, a Fourier analysis was performed on the sample set of

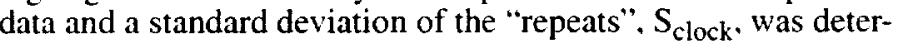

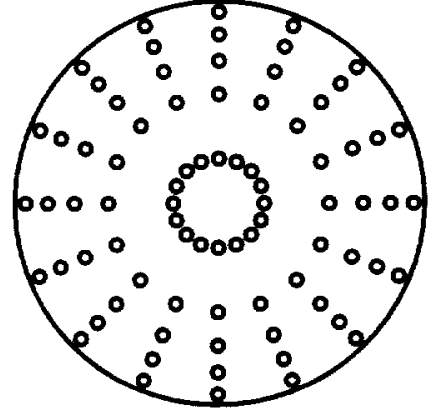

(a) 80-probe rake

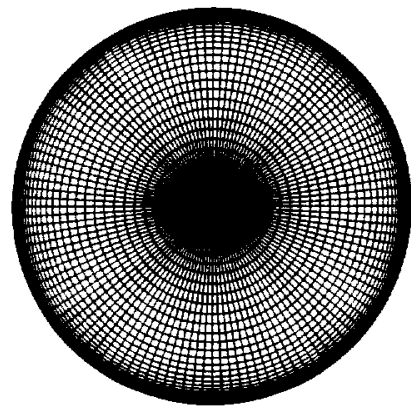

(b) Computational grid
Figure (5): Total pressure and distortion measurement arrays.

mined for each of the Fourier harmonic 1/2 amplitudes. In order to check the constant variance assumption associated with standard least square regression, a simple F-test for comparing the minimum standard deviation to the maximum standard deviation $\left(F=S^{2}{ }_{\max } / S^{2}\right.$ min $)$ was conducted for each of the five responses. The results are presented in Table (6). Since each F-test exceeded the $95 \%$ confidence critical value of $F(0.975,9,9)=4.03$, the assumption of constant variation across the design space had to be discarded. This meant that a regression technique known as weighted least squares regression had to be employed for analyzing the $10 \times 26=260$ data samples in the DOE. The weights in these regression analyses were set to $1 / \mathrm{S}^{2}$ clock.

\begin{tabular}{|c|c|c|}
\hline Response & $F=\mathrm{S}^{2}{ }_{\max } / \mathrm{S}^{2}{ }_{\min }$ & $F(0.975,9,9)$ \\
\hline $\mathrm{F} 1 / 2$ & 1939.9 & 4.03 \\
\hline $\mathrm{F} 2 / 2$ & 53.5 & 4.03 \\
\hline $\mathrm{F} 3 / 2$ & 160.0 & 4.03 \\
\hline $\mathrm{F} 4 / 2$ & 135.4 & 4.03 \\
\hline $\mathrm{FS} / 2$ & 47.6 & 4.03 \\
\hline
\end{tabular}

Table (6): Fourier Harmonic $1 / 2$ amplitude F-test compliance.

The data reduction for the inlet total pressure recovery and engine face distortion differed greatly from the harmonic analysis of distortion described. There exists no generalized methodology to evaluate the Fourier harmonic 1/2 amplitudes of engine face distortion for more than five probes in the radial direction. Hence, both the inlet total pressure recovery and engine face distortion were calculated directly from the computational grid at the engine face station. See Figure (5b). This computational mesh was composed of $49 \times 121$ grid points in the fullplane. The DC60 engine face distortion descriptor ${ }^{(8)}$ is defined such that it can be determined from either a computational grid or a standard measurement rake. It is the only recognized distortion descriptor that has this property, and hence, was chosen for this study.

\section{“Lower Order" Robust Design Methodology}

In the traditional Taguchi Robust Parameter Design methodology, a two tier experimentation strategy is used to solve the robust design problem. The control factors (design variables) are studied in their own DOE, called the inner array. A separate DOE, called the outer array, is constructed using only the environmental or noise variables. For each point in the inner array DOE, the entire outer array DOE is run and a Taguchi-style signal-to-noise ratio, $(S / N)$ is calculated using the values from the outer (mission) array matrix. The $\mathrm{S} / \mathrm{N}$ becomes the response that is analyzed over the controlled factor variables in the inner array. Using the regression model, the $\mathrm{S} / \mathrm{N}$ response is maximized/minimized to find the optimal settings for the inner array variables that produces the best response that is robust/insensitive to the outer array variables. Because the outer (mission) array matrix is run for every point in the inner design array DOE, the setup is called a product array. While a robust flow control installation can be established using the traditional Taguchi Robust Parameter Design methodology, the performance information of that installation over the outer array (mission) variable range is lost. That information gets rolled into the $S / N$, i.e. signal-to-noise parameter. Also, the traditional style Taguchi DOE is expensive. The total number of experiments is the product of the number of experiments in the inner array matrix times the number of experiments in the outer array matrix.

Cost saving can be achieved by a DOE in which the noise factors (mission variables) are introduced directly into the inner array design matrix with the controlled (design) variables. This is called a combined array format, which can have significant run-size savings over the traditional Taguchi robust design methods. Thus, in the new approach to a Robust Design methodology, a single DOE is established composed of both the inner array (design) variables and outer array (mission) variables. At each point in the combined DOE, the responses are measured. Using weighted or ordinary least squares (OLS) regression, a model is built that is a function of both the inner array (design) variables and outer array (mission) variables. For the current study, the resulting regression model for each response was a subset of the full quadratic model permitted by the DOE, namely:

$$
\begin{aligned}
Y= & \beta_{0}+\beta_{1} h_{1}+\beta_{2} h_{2}+\beta_{3} h_{3}+\beta_{4} \alpha \\
& +\beta_{11} h_{1}^{2}+\beta_{22} h_{2}^{2}+\beta_{33} h_{3}^{2}+\beta_{44} \alpha^{2} \\
& +\beta_{12} h_{1} h_{2}+\beta_{13} h_{1} h_{3}+\beta_{14} h_{1} \alpha \\
& +\beta_{23} h_{2} h_{3}+\beta_{24} h_{2} \alpha+\beta_{34} h_{3} \alpha
\end{aligned}
$$

To bring to fruition the desired robustness aspect of this study, this second order model in both the inner array and outer array was exploited in a unique way during the optimization phase. 
This method has been designated as the economical "Lower Order" approach.

For comparison purposes, consider a traditional Taguchi Robust DOE in which the number of required experiments is much higher (i.e. the number of experiments in the inner array matrix times the number of experiments in the outer array matrix). An alternate method of analyzing the data from such a DOE design is to not lose information by collapsing the outer array matrix information into a $\mathrm{S} / \mathrm{N}$, but to model the actual response data using an expanded regression model that includes the outer array variables directly. Such an approach results in the inclusion of the higher order interactive terms directly into the regression model. This approach is discussed by Anderson and Keller ${ }^{(5)}$ and has been termed the "Higher Order" approach to the analysis of a traditional Taguchi Robust DOE. In the cases studied to date, the less economical Taguchi approach using the introduction of these higher order interactions has resulted in marginal differences in the final optimal robust results when compared to the new economical combined "Lower Order" approach.

\section{Flow Control Mission Studies}

To illustrate the potential of economical Robust Design methodology, two different mission strategies were considered for the subject inlet, namely (1) Maximum Performance, and (2) Maximum HCF Life Expectancy. The Maximum Performance mission sought to minimize the inlet duct losses (maximize the engine face total pressure recovery) subject to the constraint that the DC60 engine face distortion be less than 0.10 , while no conditions were placed on the first five Fourier harmonic $1 / 2$ amplitudes of distortion. A DC60 distortion level of 0.10 or less is significant because it would be acceptable for a commercial engine application. The Maximum HCF Life Expectancy mission sought to minimize the mean of the first five Fourier harmonic 1/ 2 amplitudes, also subject to the constraint that the DC60 engine face distortion be less than 0.10. In this mission, however, no constraint was placed upon the inlet total pressure recovery. For each of these missions strategies, both an "Optimal Robust" (open loop control) and an "Optimal Adaptive" (closed loop control) installation were designed using the angle-of-incidence from 0 to $20^{\circ}$ as the noise factor. The "Optimal Robust" installation used economical Robust Design methodology to arrive at a single design which operated over the entire angle-of-incident range (open loop control). The "Optimal Adaptive" installation optimized all the design parameters at each angle-of-incidence within the search (optimization) routine. Thus, in the "Optimal Adaptive" system, the factor values were adjusted at each angle of attack to simulate a closed loop system. Thus the "Optimal Adaptive" installation would require a closed loop control system to sense a proper signal for each effector and modify that effector device, whether mechanical or fluidic, for optimal inlet performance.

Maximum Performance Mission - Specifically, for the "Optimal Adaptive" Maximum Performance MSFC installation, at each angle-of-incidence, the engine face distortion constraint DC $60 \leq 0.10$ was imposed and a search was made over the design variable space to locate that installation geometry that maximized PFAVE. For this mission, no constraints were imposed upon the first five Fourier harmonic $1 / 2$ amplitudes of engine face distortion. In a similar manner, for the "Optimal Robust" MSFC installation, the engine face distortion constraint DC $60 \leq 0.10$ was imposed and a search was made over the design variable space to locate that installation geometry that maximized the optimization parameter $\Sigma Y_{\alpha} / \mathrm{m}$, where $\mathrm{Y}_{\alpha}=$ PFAVE at each of the $\alpha=0.0$, $1.0, \ldots, 20.0^{\circ}$ angle-of-incidences and $m=21$. Again, for this mission no constraints were placed on the first five Fourier harmonic $1 / 2$ amplitudes of engine face distortion. This procedure economically defined one installation that was "Optimal Robust" over the entire range of angle-of-incidence.

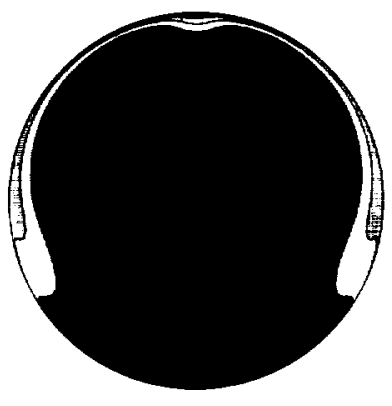

(a) Total Pressure

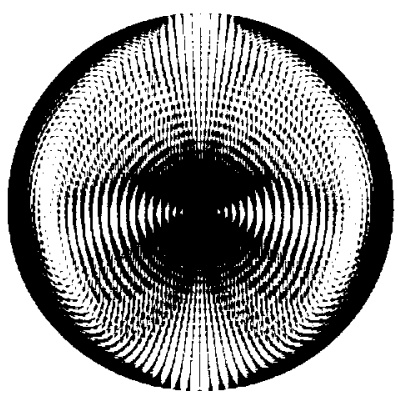

(b) Velocity Vectors
Figure (6): "Optimal Robust" Maximum Performance MSFC installation engine face CFD solution, $\alpha=0.0^{\circ}$.

\begin{tabular}{|c|c|c|}
\hline Factor/Response & Range/Constraint & Optimal Value \\
\hline$h_{1}$ & 0.0 to 2.0 & 0.0 \\
\hline$h_{2}$ & 0.0 to 2.0 & 0.0 \\
\hline$h_{3}$ & 0.0 to 2.0 & 1.90 \\
\hline PFAVE & Maximized & 0.97329 \\
\hline DC60 & $\leq$ 0.10 & 0.08401 \\
\hline F1/2 & Unconstrained & 0.00705 \\
\hline F2/2 & Unconstrained & 0.01636 \\
\hline F3/2 & Unconstrained & 0.01651 \\
\hline F4/2 & Unconstrained & 0.00527 \\
\hline F5/2 & Unconstrained & 0.00106 \\
\hline FM/2 & Unconstrained & 0.00925 \\
\hline
\end{tabular}

Table (8): "Optimal Robust" Maximum Performance MSFC installation inlet CFD performance, $\alpha=0.0^{\circ}$.

The "Optimal Robust" Maximum Performance installation was determined to have the following effector vane heights $(\mathrm{mm}): \mathrm{h}_{1}=0.0, \mathrm{~h}_{2}=0.0, \mathrm{~h}_{3}=1.9$. The inlet CFD solution for the "Optimal Robust" Maximum Performance installation design at the engine face is presented in Figure (6) and the CFD performance of the inlet with that installation is shown in Table (8). The near wall streamlines for the baseline solution and the "Optimal Robust" Maximum Performance installation design are presented in Figures (7) and (8) respectively. A comparison of these two figures indicates the underlying operational purpose of micro- 
scale secondary flow control. In the baseline case presented in Figure (7), the flow in a very thin layer adjacent to the walls "over turns" as a result of a loss of momentum within the inlet boundary layer. Eventually, this "over-turning" will cause a vortex pair to form in the inlet passage. This vortex pair results in total pressure loss and severe total pressure distortion at the engine face. It is not necessary for this vortex to "lift-off" or separate from the walls for high total pressure loss and distortion to occur (hence the terminology inlet "secondary flow control" rather than "separation control"). By introducing the micro-actuators into the inlet, whether vanes or jets, the "over-turning" in the inlet boundary is prevented. See Figure (8). Consequently, the passage vortex will

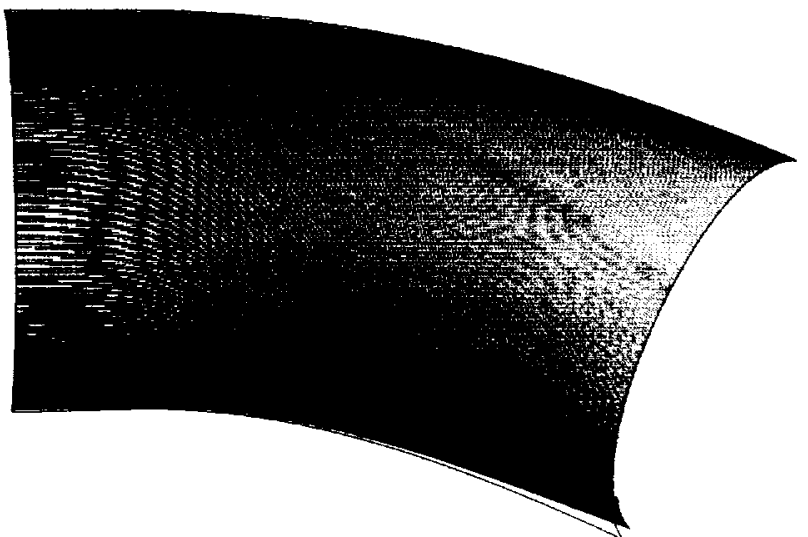

Figure (7): Near wall streamlines within effector region, baseline CFD solution, $\alpha=0.0^{\circ}$.

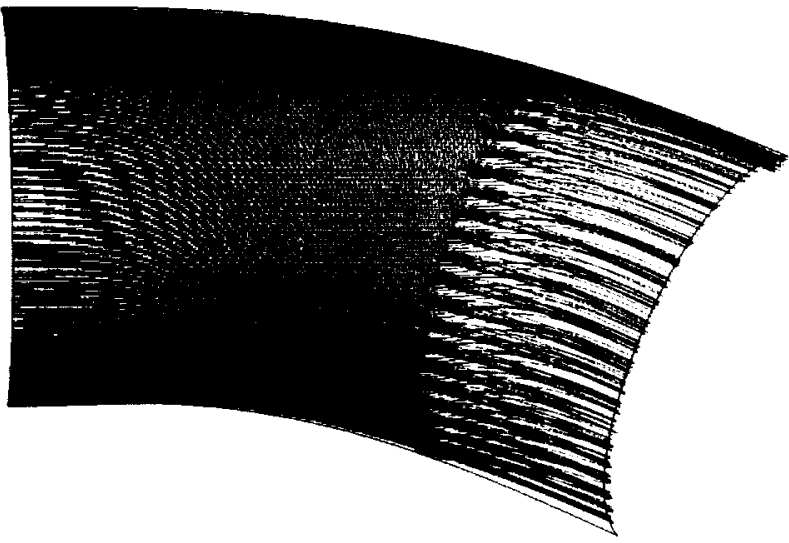

Figure (8): Near wall streamlines within effector region, "Optimal Robust" Maximum Performance MSFC installation design, $\alpha=0.0^{\circ}$.

not form or, at worst, is greatly reduced in strength, which will result in a vast improvement in engine face distortion. Therefore, the entire inlet flow field can be managed by controlling the secondary flow in a thin layer adjacent to the inlet walls. In the MASC concept, micro-scale actuation is used as an approach called "secondary flow control" to alter the S-duct's inherent secondary flow characteristics with the goal of simultaneously improving the critical system level performance metrics pressure recovery, engine face distortion, and HCF characteristics.

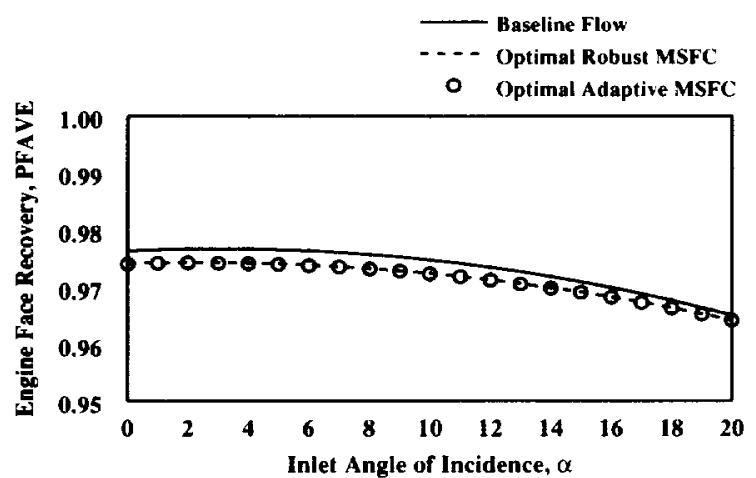

(a) Total Pressure Recovery Characteristics.

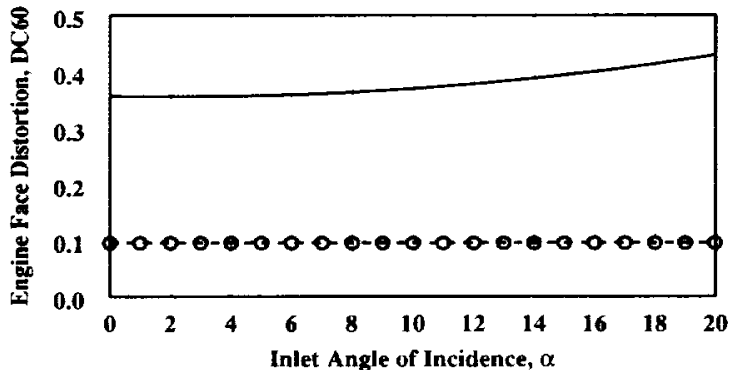

(b) Engine Face Distortion Characteristics.

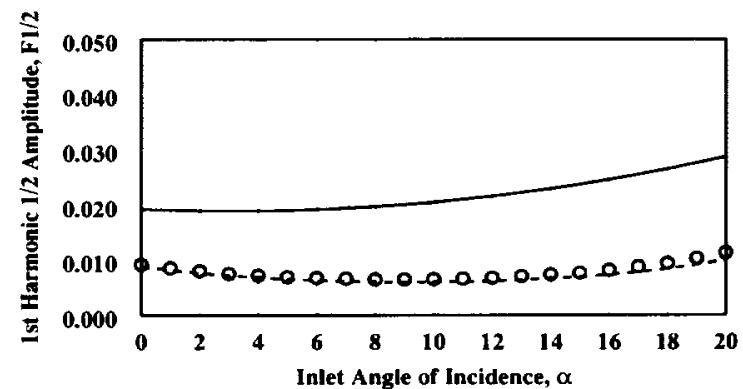

(c) 1st Fourier Harmonic $1 / 2$ Amplitude Characteristics.

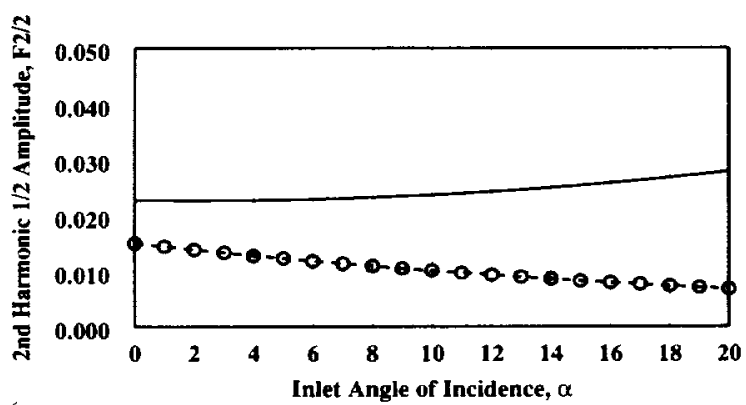

(d) 2nd Fourier Harmonic 1/2 Amplitude Characteristics.

Figure (9): Comparison between "Optimal Robust" and "Optimal Adaptive" Maximum Performance MSFC installation designs. 


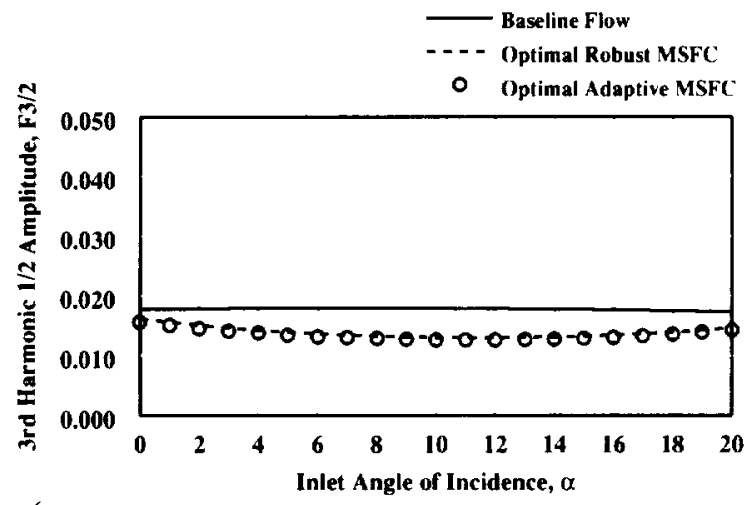

(e) 3th Fourier Harmonic 1/2 Amplitude Characteristics.

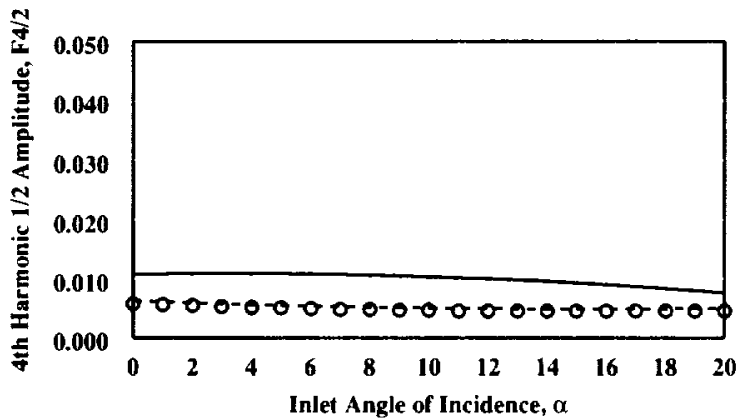

(f) 4th Fourier Harmonic 1/2 Amplitude Characteristics.

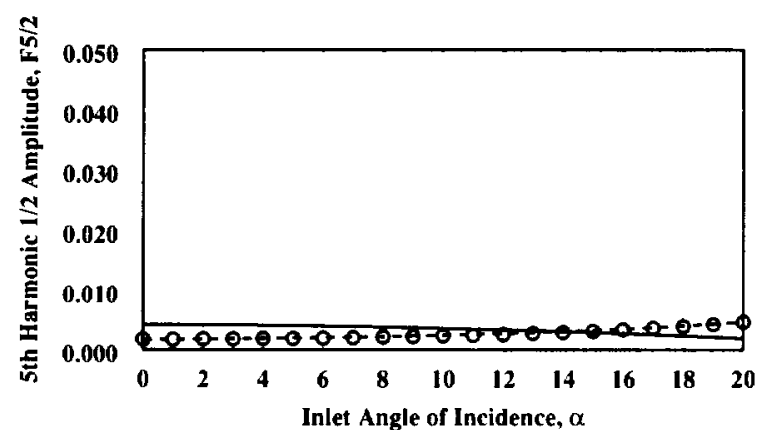

(g) 5th Fourier Harmonic $1 / 2$ Amplitude Characteristics.

Figure (9): Comparison between "Optimal Robust" and "Optimal Adaptive" Maximum Performance MSFC installation designs, cont.

Comparisons between the performance results of the "Optimal Robust" and "Optimal Adaptive" installations for the Maximum Performance inlet mission are shown in Figures (9a) to $(9 \mathrm{~g})$. Also presented in Figures $(9 \mathrm{a})$ to $(9 \mathrm{~g})$ are the baseline flow solutions, i.e. the inlet performance without flow control, for each response. It is apparent from Figure (9a) that flow control was unable to increase total pressure recovery above the baseline flow. A recovery improvement could not be demonstrated for this case. The losses resulting from the micro-vanes actuators, which were included in the CFD analysis, were somewhat greater than improved efficiency of the flow process as a result of the vane installation. This is not always the case, since experimental evidence exists that clearly demonstrates that substantial improvement in recovery is achievable under severe vortex lift-off conditions. ${ }^{(2)}$ It is important, however, that a vane model in a full Navier-Stokes includes a loss term associated with the microvanes.

Essentially, the "Optimal Robust" and "Optimal Adaptive" installations were the same configuration based on their performance over the angle-of-incidence range of 0 to $20^{\circ}$. This is not surprising, since there exists experimental data ${ }^{(9)}$ that demonstrate that a fixed secondary flow control installation can provide essentially the same low DC60 distortion level, i.e. DC60 $\leq 0.10$, over a substantial angle-of-incidence range. Secondary flow control in inlets is inherently robust, provided it is optimally designed. By comparing Figure $(9 \mathrm{c})$ with Figure $(9 \mathrm{e})$ an interesting feature of this mission can be seen. There was the shift in the peak amplitude from the first Fourier harmonic 1/2 amplitude to the third Fourier harmonic $1 / 2$ amplitude as a result of secondary flow control. With micro-scale secondary flow control, a dominant "\# per rev" frequency may thus be shifted to avoid the natural resonance frequency of the engine fan blades. This shift is usually to the higher "\# per rev" frequency range.

For the "Optimal Adaptive" Maximum HCF Life Expectancy MSFC installation, at each angle-of-incidence, the engine face distortion constraint, DC60 $\leq 0.10$, was imposed and a search was made over the design variable space to locate that installation geometry that minimized $\Sigma \mathrm{Fj} / 5$, the mean of the first five Fourier harmonic $1 / 2$ amplitudes present in the engine face distortion profile. For this mission, no constraint was placed on the inlet total pressure recovery.

Maximum HCF Life Expectancy Mission - In a similar manner, for the "Optimal Robust" Maximum HCF Life Expectancy MSFC installation, the engine face distortion constraint, DC60 $\leq 0.10$, was imposed and a search was made over the design variable space to locate that installation geometry that minimized $\Sigma Y_{\alpha}{ }^{2} / \mathrm{m}$, where $\mathrm{Y}_{\alpha}=\Sigma F j / 5$, the mean of the first five Fourier harmonic $1 / 2$ amplitudes present in the engine face distortion profile at each of the $\alpha=0.0,1.0, \ldots, 20.0^{\circ}$ angle-of-incidence and $m=21$. Again for this mission, no constraints were placed on the inlet total pressure recovery. This procedure economically defined one installation that was "Optimal Robust" over the range of angle-of-incidence.

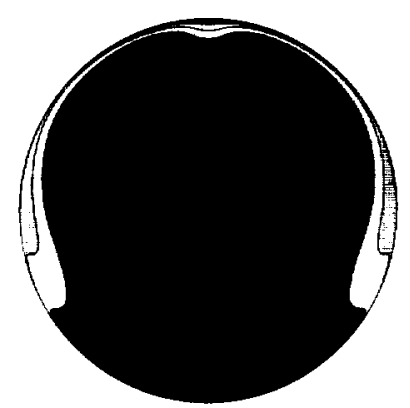

(a) Total Pressure

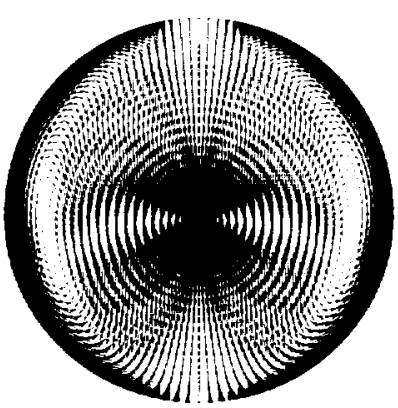

(b) Velocity Vectors
Figure (10): "Optimal Robust" Maximum HCF Life Expectancy MSFC installation engine face CFD solution, $\alpha=0.0^{\circ}$. 


\begin{tabular}{|c|c|c|}
\hline Factor/Response & Range/Constraint & Optimal Value \\
\hline$h_{1}$ & 0.0 to 2.0 & 0.0 \\
\hline$h_{2}$ & 0.0 to 2.0 & 0.0 \\
\hline$h_{3}$ & 0.0 to 2.0 & 2.00 \\
\hline PFAVE & Unconstrained & 0.97377 \\
\hline DC60 & $\leq 0.10$ & 0.08216 \\
\hline F1/2 & Minimized & 0.00708 \\
\hline F2/2 & Minimized & 0.01517 \\
\hline F3/2 & Minimized & 0.01583 \\
\hline F4/2 & Minimized & 0.00523 \\
\hline F5/2 & Minimized & 0.00102 \\
\hline FM/2 & Minimized & 0.00887 \\
\hline
\end{tabular}

Table (9): "Optimal Robust" Maximum HCF Life Expectancy MSFC installation inlet CFD performance, $\alpha=0.0^{\circ}$.

The "Optimal Robust" Maximum HCF Life Expectancy installation design was also determined through a search process to have the following effector vane heights and chord lengths $(\mathrm{mm}): \mathrm{h}_{1}=0.0, \mathrm{~h}_{2}=0.0, \mathrm{~h}_{3}=2.0$. The inlet CFD solution for the "Optimal Robust" Maximum HCF Life Expectancy installation design at the engine face is presented in Figure (10) and the performance of the inlet with this installation is presented in Table (9).

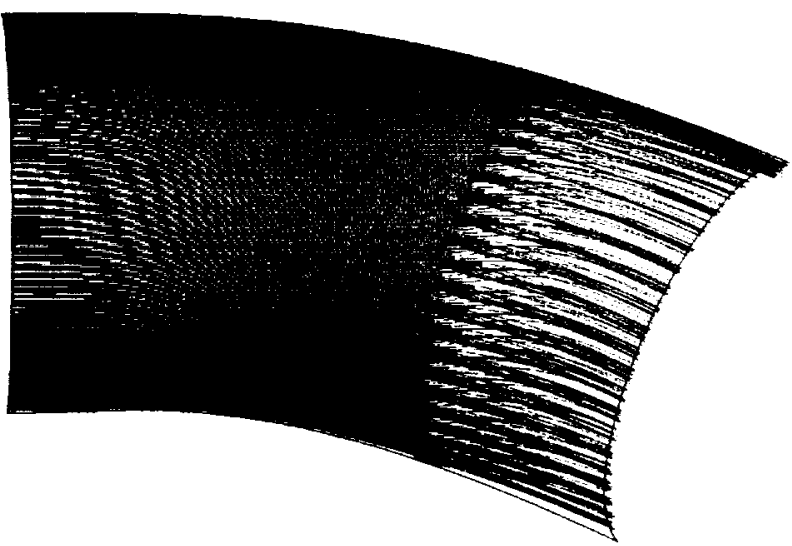

Figure (11): Near wall streamlines within effector region, "Optimal Robust" Maximum HCF Life Expectancy MSFC installation design, $\alpha=0.0^{\circ}$.

Presented in Figure (11) is the near wall streamlines for the "Optimal Robust" Maximum HCF Life Expectancy installation design for $\alpha=0.0^{\circ}$. Again, notice the effect of the microvane actuators in preventing the over-turning of the flow adjacent

to the inlet walls and thus suppressing the passage vortex formation. Once again, there was a vast improvement in engine face distortion. Figures(12a) through Figure (12g) shows that the differences between the "Optimal Adaptive" and "Optimal Robust" secondary flow installation design were again minimal for the Maximum HCF Life Expectancy mission. Requiring the mean of the first five Fourier harmonic $1 / 2$ amplitudes present in the engine face distortion profile to be minimized does not necessarily mean that all the amplitudes will be reduced relative to the baseline flow. Figures (12c) through (12f) show that the first through the fourth Fourier $1 / 2$ harmonic amplitude all decreased relative to the baseline flow. However, the fifth Fourier harmonic 1/2 amplitude increased above the baseline case at the higher angle-of-incidences. See Figure $(12 \mathrm{~g})$. The important conclusion is that both the "Optimal Adaptive" and "Optimal Robust" mission strategies resulted in installation designs that are able to reduce the Fourier harmonic $1 / 2$ amplitudes of distortion, while maintaining a very low engine face DC60 distortion.

By comparing the "Optimal Robust" Maximum Performance installation design shown in Table (8) and the Maximum HCF Life Expectancy installation shown in Table (9), it can be seen that the two optima are almost identical $\left(h_{3}=1.90\right.$ versus $h_{3}$ $=2.0$ ). This will not in general be the case in all future studies. The different approaches to optimization can (and most probability) lead to different optima.

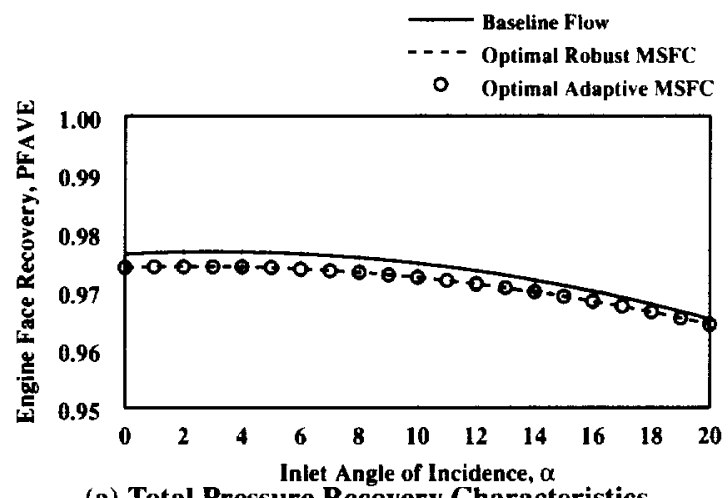

(a) Total Pressure Recovery Characteristics.

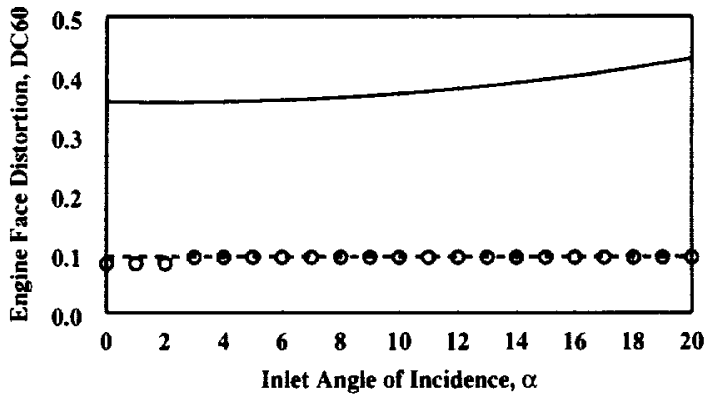

(b) Engine Face Distortion Characteristics.

Figure (12): Comparison between "Optimal Robust" and "Optimal Adaptive" Maximum HCF Life Expectancy MSFC installation designs. 


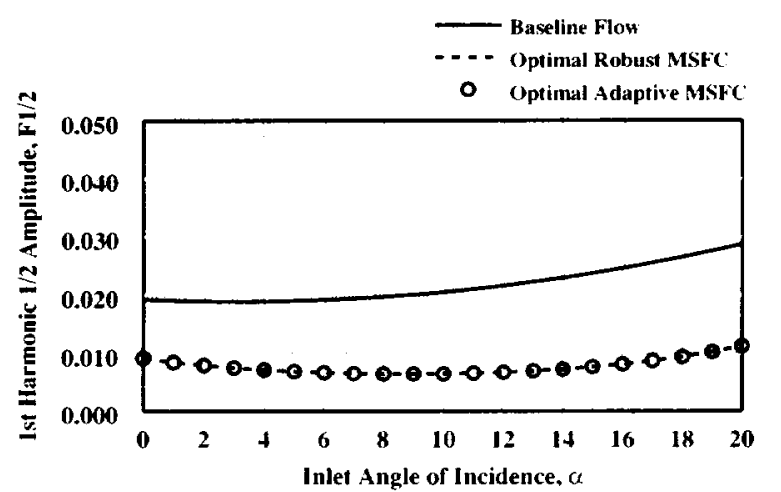

(c) 1st Fourier Harmonic 1/2 Amplitude Characteristics.

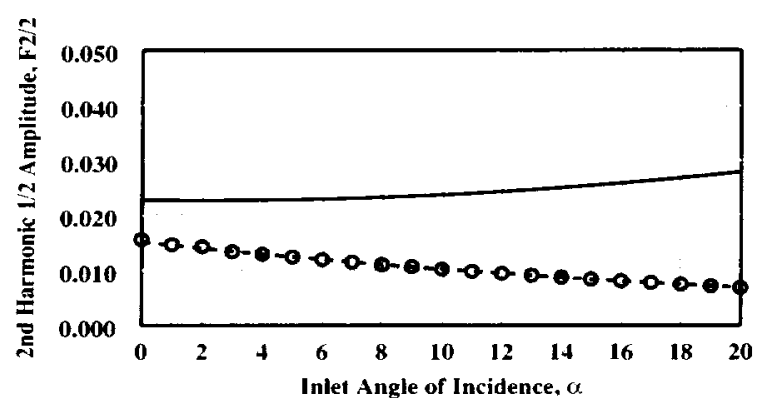

(d) 2nd Fourier Harmonic 1/2 Amplitude Characteristics.

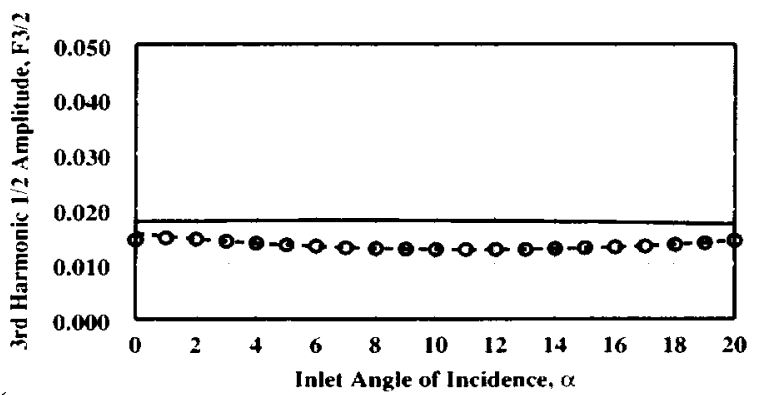

(e) 3rd Fourier Harmonic 1/2 Amplitude Characteristics.

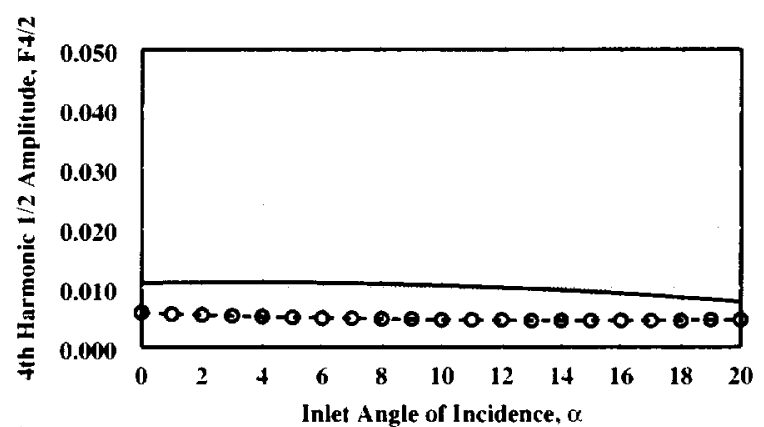

(f) And Fourier Harmonic 1/2 Amplitude Characteristics.

Figure (12): Comparison between "Optimal Robust" and "Optimal Adaptive" Maximum HCF Life Expectancy MSFC installation designs, cont.

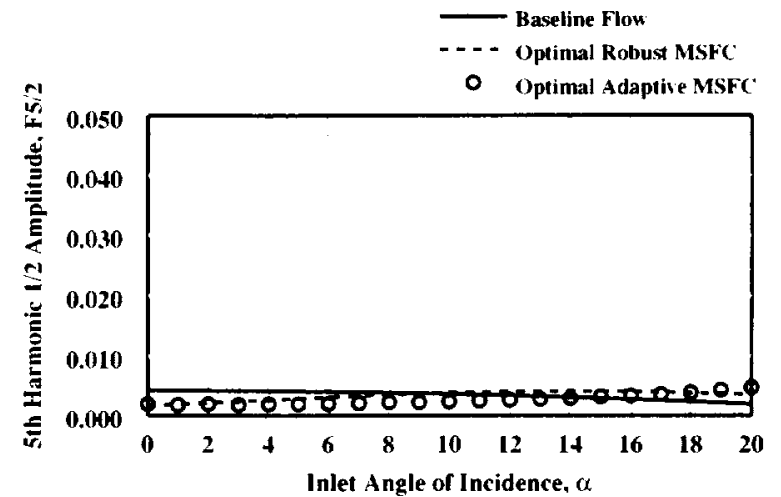

(c) 5st Fourier Harmonic 1/2 Amplitude Characteristics.

Figure (12): Comparison between "Optimal Robust" and "Optimal Adaptive" Maximum HCF Life Expectancy MSFC installation designs, cont.

\section{CONCLUSIONS}

The fundamental importance of Genichi Taguchi's contribution to Total Quality Design over traditional design approaches lies in the idea that process and product variability can be incorporated into the optimal statistical Design-of-Experiment and subsequent analysis of data. To the aerodynamicist. it represents a major breakthrough in the area of aerodynamic design of inlets, since the effect of the mission variables can be directly introduced into the design process. The inlet system can now be designed to operate with optimal performance over a range of specified mission variables. Taguchi"s Robust Parameter Design method, however, may not be optimal in the design of secondary flow installations for inlet systems because: (a) it loses information vital to the aerodynamicist and, (b) it is costly. Fortunately, the important aspects surrounding Taguchi's approach to Robust Parameter Design can and have been incorporated into an alternate economical approach and adapted to the inlet design problem. This alternate inlet design method. using a combined array approach to economical Robust Design. had significant run size savings over a traditional Taguchi approach, i.e. $26 \mathrm{CFD}$ experiments as compared to $45 \mathrm{CFD}$ experiments.

To illustrate the potential of economical Robust Design methodology. two different mission strategies were considered for the subject inlet, namely (1) Maximum Performance, and (2) Maximum HCF Life Expectancy. The Maximum Performance mission maximized total pressure recovery while the Maximum HCF Life Expectancy mission minimized the mean of the first five Fourier harmonic 1/2 amplitudes. i.e. "collectively" reduced all the harmonic 1/2 amplitudes of engine face distortion. Each of the mission strategies was subject to a low engine face distortion constraint of DC60 $\leq 0.10$, a level acceptable for commercial engines. For each of these missions strategies, an "Optimal Robust" (open loop control) and an "Optimal Adaptive" (closed loop control) installation was designed over a twenty degree angle-of-incidence range. The "Optimal Robust" installation used a new economical approach to Robust installation design to arrive at a single installation which operated over the entire angle-ofincident range (open loop control). The "Optimal Adaptive" installation optimized all the design parameters at each angle-ofincidence. Thus the "Optimal Adaptive" installation would 
require a closed loop control system to sense a proper signal for each effector and modify that effector device, whether mechanical or fluidic, for optimal inlet performance.

In general, the performance differences between the "Optimal Adaptive" and "Optimal Robust" installation designs were found to be marginal. This suggests, however, that "Optimal Robust" open loop installation designs can be very competitive with "Optimal Adaptive" close loop designs. Secondary flow control in inlets is inherently robust, provided it is optimally designed. Therefore, the new methodology presented in this paper, combined array "Lower Order" approach to Robust DOE, offers the aerodynamicist a very viable and economical way of exploring the concept of Robust inlet design, where the mission variables are brought directly into the inlet design process and insensitivity or robustness to the mission variables becomes a design objective.

\section{REFERENCES}

(1) Anderson, B. H., Miller, D. M., Yagel, P. J, and Traux, P. P., "A Study of MEMS Flow Control for the Management of Engine Face Distortion in Compact Inlet Systems", FEDSM99-6920,3rd ASME/JSME Joint Fluids Engineering Conference, San Francisco, CA. July 18-23, 1999.

(2) Hamstra, J. W., Miller, D. N., Truax, P. P., Anderson, B. H.. and Wendt, B. J., "Active Inlet Flow Control Technology Demonstration", ICAS-2000-6.11.2, 22nd International Congress of the Aeronautical Sciences, Harrogate, UK, August 27th-September $1 \mathrm{st}, 2000$.

(3) Taguchi, G and Wu, Y, "Introduction to Off-Line Quality Control," Central Quality Control Association, 1980.

(4) Anderson, B. H. and Keller, D. J., "Optimal Micro-Scale Secondary Flow Control for the Management of HCF and Distortion in Compact Inlet Diffusers", Proposed NASA TM, 2001.
(5) Anderson, B. H. and Keller, D. J., "Robust Design Methodology for Optimal MicroScale Secondary Flow Control in Compact Inlet Diffusers", NASA/TM- 2001-211278.

${ }^{16)}$ Anderson, B. H. and Keller, D. J., "Considerations in the Measurements of Engine Face Distortion for High Cycle Fatigue in Compact Inlet Diffusers". Proposed NASA TM, 2001.

(7) Willmer, A. C.. Brown, T. W. and Goldsmith, E. L., "Effects of Intake Geometry on Circular Pitot Intake Performance at Zero and Low Forward Speeds", Aerodynamics of Power Plant Installation, AGARD CP301, Paper 5, Toulouse, France, May 1981, pp $51-56$.

${ }^{(8)}$ Goldsmith, E. L. and Seddon, J. (eds), "Practical Intake Aerodynamics," Blackwell Scientific Publications, Oxford, 1993.

(9) Bender, E. E, Anderson, B. H., and Yagle. P. J., "Vortex Generator Modeling for Navier Stokes Code", FEDSM99-69219, 3rd ASME/JSME Joint Fluids Engineering Conference, San Francisco, CA, July 18-23, 1999.

(10) Gibb, J. and Anderson, B. H., "Vortex Flow Control Applied to Aircraft Intake Ducts," Proceedings of the Royal Aeronautical Society Conf., High Lift and Separation Control, Paper No. 14, Bath, UK, March, 1995.

(11) Ludwig, G. R., "Aeroelastic Considerations in the Measurement of Inlet Distortion", 3rd National Turbine Engine High Cycle Fatigue Conference, 1998. 
Public reporting burden for this collection of information is estimated to average 1 hour per response, including the time for reviewing instructions, searching existing data sources. gathering and maintaining the data needed. and completing and reviewing the collection of information. Send comments regarding this burden estimate or any other aspect of this collection of information, including suggestions for reducing this burden, to Washington Headquarters Services. Directorate for Information Operations and Reports. 1215 Jefferson Davis Highway. Suite 1204, Arlington, VA 22202-4302, and to the Office of Management and Budget. Paperwork Reduction Project (0704-0188). Washington. DC 20503.

\begin{tabular}{|l|l|l|}
\hline 1. AGENCY USE ONLY (Leave blank) & 2. REPORT DATE & 3. REPORT TYPE AND DATES COVERED
\end{tabular}

\begin{tabular}{|l|l|l|}
\hline & December 2001 & Technical Memorandum \\
\hline
\end{tabular}

\section{TITLE AND SUBTITLE}

5. FUNDING NUMBERS

A Robust Design Methodology for Optimal Microscale

Secondary Flow Control in Compact Inlet Diffusers

6. AUTHOR(S)

Bernhard H. Anderson and Dennis J. Keller

7. PERFORMING ORGANIZATION NAME(S) AND ADDRESS(ES)

National Aeronautics and Space Administration

John H. Glenn Research Center at Lewis Field

Cleveland, Ohio 44135-3191

WU-708-5.3-1.3-00

9. SPONSORING/MONITORING AGENCY NAME(S) AND ADDRESS(ES)

National Aeronautics and Space Administration

Washington, DC 20546-0001

8. PERForming ORGaNIZATION

REPORT NUMBER

E-1.3077

10. SPONSORINGMONITORING

AGENCY REPORT NUMBER

11. SUPPLEMENTARY NOTES

Prepared for the 40th Aerospace Sciences Meeting and Exhibit sponsored by the American Institute of Aeronautics and Astronautics. Reno, Nevada, January 14-17, 2002. Bemhard H. Anderson. NASA Glenn Research Center, and Dennis J. Keller, RealWorld Quality Systems, 20388 Bonnie Bank Boulevard, Rocky River, Ohio 441 16. Responsible person, Bernhard H. Anderson, organization code 5850, 216-433-5822.

12a. DISTRIBUTION/AVAILABILITY STATEMENT

12b. DISTRIBUTION CODE

Unclassified - Unlimited

Subject Category: 07

Distribution: Nonstandard

Available electronically at hip://glirs.grc.nasa.gov/GLTRS

This publication is available from the NASA Center for AeroSpace Information. 301-621-0390.

13. ABSTRACT (Maximum 200 words)

It is the purpose of this study to develop an economical Robust design methodology for microscale secondary flow control in compact inlet diffusers. To illustrate the potential of economical Robust Design methodology, two different mission strategies were considered for the subject inlet, namely ( 1 ) Maximum Performance, and (2) Maximum HCF Life Expectancy. The Maximun Perfomance mission maximized total pressure recovery while the Maximum HCF Life Expectancy mission minimized the mean of the first five Fourier harmonic amplitudes. i.e.. "collectively" reduced all the harmonic $1 / 2$ amplitudes of engine face distortion. Each of the mission strategies was subject to a low engine face distortion constraint, i.e.. DC $60 \leq$ 0.10 . which is a level acceptable for commercial engines. For each of these missions strategies. an "Optimal Robust" (open loop control) and an "Optimal Adaptive" (closed loop control) installation was designed over a rwenty degree angle-of-incidence range. The "Optinal Robust" installation used economical Robust Design methodology to arrive at a single design which operated over the entire angle-of-incident range (open loop control). The "Optimal Adaptive" installation optimized all the design parameters at each angle-of-incidence. Thus. the "Optimal Adaptive" installation would require a closed loop control system to sense a proper signal for each effector and modify that effector device, whether mechanical or fluidic, for optimal inlet performance. In general, the performance differences between the "Optimal Adaptive" and "Optimal Robust" installation designs were found to be marginal. This suggests, however, that "Optimal Robust" open loop installation designs can be very competitive with "Optimal Adaptive" close loop designs. Secondary flow control in inlets is inherently robust, provided it is optimally designed. Therefore, the new methodology presented in this paper. combined array "Lower Order" approach to Robust DOE, offers the aerodynamicist a very viable and economical way of exploring the concept of Robust inlet design, where the mission variables are brought directly into the inlet design process and insensitivity or robustness to the mission variables becomes a design objective.

14. SUBJECT TERMS

Propulsion; Flow control

15. NUMBER OF PAGES

18

\begin{tabular}{|c|c|}
\hline $\begin{array}{c}\text { 17. SECURITY CLASSIFICATION } \\
\text { OF REPORT } \\
\text { Unclassified }\end{array}$ & $\begin{array}{c}\text { 18. SECURITY CLASSIFICATION } \\
\text { OF THIS PAGE } \\
\text { Unclassified }\end{array}$ \\
\hline
\end{tabular}
19. SECURITY CLASSIFICATION OF ABSTRACT

16. PRICE CODE

\begin{tabular}{|c|c|}
\hline & $\begin{array}{c}\text { 15. NUMBER OF PAGES } \\
18\end{array}$ \\
\hline & 16. PRICE CODE \\
\hline $\begin{array}{l}\text { 19. SECURITY CLASSIFICATION } \\
\text { OF ABSTRACT } \\
\text { Unclassified }\end{array}$ & 20. LIMITATION OF ABSTRACT \\
\hline
\end{tabular}

NSN $7540-01-280-5500$

Standard Form 298 (Rev. 2-89)

Prescribed by ANSI Std. Z39-18 298-102 
